H. Morikawa

Nagoya Math. J.

Vol. 99 (1985), 45-62

\title{
SOME RESULTS ON HARMONIC ANALYSIS ON COMPACT QUOTIENTS OF HEISENBERG GROUPS
}

\author{
HISASI MORIKAWA
}

Heisenberg group $H_{2 g+1}(R)$ of dimension $2 g+1$ is a real nilpotent group defined on $\boldsymbol{R} \times \boldsymbol{R}^{g} \times \boldsymbol{R}^{g}$ by the law of composition

$$
\left(x_{0}, \hat{x}, x\right) \circ\left(y_{0}, \hat{y}, y\right)=\left(x_{0}+y_{0}+\hat{x}^{t} y, \hat{x}+\hat{y}, x+y\right),
$$

which is isomorphic to the unipotent matrix group

$$
\left\{\left(\begin{array}{cccc}
1 & \hat{c}_{1}, \cdots, \hat{c}_{g}, & c_{0} \\
& 1 & & c_{1} \\
& \ddots & \vdots \\
& & 1 & c_{g} \\
& & & 1
\end{array}\right\} \quad\left(c_{0}, \hat{c}_{i}, c_{i} \in R, 1 \leqslant i, j \leqslant g\right) .\right.
$$

$H_{2 g+1}(Z)$ means the discrete subgroup of integral elements, and $L^{2}\left(H_{2 g+1}(Z) \backslash\right.$ $\left.H_{2 g+1}(R)\right)$ is the $L^{2}$-space of the quotient space

$$
H_{2 g+1}(Z) \backslash H_{2 g+1}(R)
$$

with respect to the invariant measure

$$
d x_{0} d \hat{x} d x=d x_{0} d \hat{x}_{1} \ldots d \hat{x}_{g} d x_{1} \cdots d x_{g} .
$$

The right action of $H_{2 g+1}(R)$ induces a unitary representation $\rho$ on $L^{2}\left(H_{2 g+1}(Z) \backslash H_{2 g+1}(R)\right)$ :

$$
\rho\left(y_{0}, \hat{y}, y\right) \phi\left(x_{0}, \hat{x}, x\right)=\phi\left(\left(x_{0}, \hat{x}, x\right) \circ\left(y_{0}, \hat{y}, y\right)\right) .
$$

For each non-zero real number $\lambda H_{2 g+1}(R)$ also acts on the usual $L^{2}$ space $L^{2}\left(\boldsymbol{R}^{g}\right)$ as follows

$$
\begin{array}{r}
\chi_{\lambda}\left(y_{0}, \hat{y}, y\right) f(\xi)=\exp \left(2 \pi \lambda \sqrt{-1}\left(y_{0}+\hat{y}^{t} \xi\right)\right) f(\xi+y), \\
\left(f(\xi) \in L^{2}\left(\boldsymbol{R}^{g}\right), \quad\left(y_{0}, \hat{y}, y\right) \in H_{2 g+1}(R)\right),
\end{array}
$$

Since Lebesgue measure is invariant with respect to translations, $\chi_{\lambda}$ is a Received March 16, 1984. 
unitary representation of $H_{2 g+1}(\boldsymbol{R})$.

In the present article, for each theta function $\vartheta(\tau \mid z)$ of level $n$, we shall construct a transformation

$$
\phi_{\vartheta}: L^{2}\left(\boldsymbol{R}^{g}\right) \longrightarrow L^{2}\left(H_{2 g+1}(Z) \backslash H_{2 g+1}(R)\right)
$$

such that

i) $\left\langle\phi_{\vartheta}\left(f_{1}\right), \phi_{\vartheta}\left(f_{2}\right)\right\rangle=\left\langle f_{1}, f_{2}\right\rangle\left(f_{1}, f_{2} \in L^{2}\left(\boldsymbol{R}^{g}\right)\right)$

ii) $\phi_{\vartheta} \circ \chi_{n}\left(y_{0}, \hat{y}, y\right)=\rho\left(y_{0}, \hat{y}, y\right) \circ \phi_{\vartheta}\left(\left(y_{0}, \hat{y}, y\right) \in H_{2 g+1}(\boldsymbol{R})\right)$

$\phi_{\vartheta}$ is actually given by

$$
\begin{aligned}
\phi_{9}\left(\exp \left(\pi n \sqrt{-1}\left(\xi \tau^{t} \xi\right)\right) \xi^{j}\right)\left(x_{0}, \hat{x}, x\right) & \\
= & (2 \pi n \sqrt{-1})^{-|j|} \exp \left(\pi n \sqrt{-1}\left(x \tau^{t} x+2 x^{t} x-2 x_{0}\right)\right) \\
& \left(2 \pi n \sqrt{-1}+\frac{\partial}{\partial \hat{x}}\right)^{j} \vartheta(\tau \mid \hat{x}+x \tau) .
\end{aligned}
$$

Choosing the canonical basis of theta functions

$$
\vartheta^{(n)}\left[\begin{array}{c}
a / n \\
0
\end{array}\right](\tau \mid z) \quad\left(a \in Z^{g} / n Z^{g}, n \geqq 1\right),
$$

we denote by $\phi_{\tau}^{(n)}\left[\begin{array}{c}a / n \\ 0\end{array}\right]$ the transformation

$$
L^{2}\left(\boldsymbol{R}^{g}\right) \longrightarrow L^{2}\left(H_{2 g+1}(Z) \backslash H_{2 g+1}(R)\right)
$$

associating with theta function $\vartheta^{(n)}\left[\begin{array}{c}a / n \\ 0\end{array}\right](\tau \mid z)$ and denote

$$
\boldsymbol{H}^{(n)}\left[\begin{array}{c}
a / n \\
0
\end{array}\right]=\phi_{\tau}^{(n)}\left[\begin{array}{c}
a / n \\
0
\end{array}\right]\left(L^{2}\left(\boldsymbol{R}^{g}\right)\right), \quad \overline{\boldsymbol{H}^{(n)}\left[\begin{array}{c}
a / n \\
0
\end{array}\right]}=\overline{\phi_{\tau}^{(n)}\left[\begin{array}{c}
a / n \\
0
\end{array}\right]\left(L^{2}\left(\boldsymbol{R}^{g}\right)\right) .}
$$

Then the decomposition of the unitary representation $\rho$ is given by

$$
\begin{aligned}
& L^{2}\left(H_{2 g+1}(\boldsymbol{Z}) \backslash \boldsymbol{H}_{2 g+1}(\boldsymbol{R})\right)=\left(\underset{\substack{a \in \boldsymbol{Z}^{8}, n \boldsymbol{Z}^{g} \\
n \geqq 1}}{\bigoplus} \boldsymbol{H}^{(n)}\left[\begin{array}{c}
a / n \\
0
\end{array}\right]\right) \\
& \oplus\left(\underset{\substack{a \in Z^{g} / n Z^{g} \\
n \geqq 1}}{\boldsymbol{H}_{\tau}^{(n)}\left[\begin{array}{c}
a / n \\
0
\end{array}\right]}\right) \oplus\left(\underset{\substack{\hat{k}, k) \in Z^{g} \times \mathbf{Z}^{g} \\
\bigoplus^{g}}}{ } C \exp \left(2 \pi n \sqrt{-1}\left(\hat{k}^{t} \hat{x}+k^{t} x\right)\right)\right) .
\end{aligned}
$$

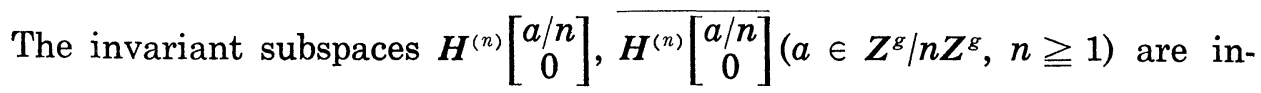
dependent of the choice of $\tau$, and $\boldsymbol{H}^{(n)}\left[\begin{array}{c}a / n \\ 0\end{array}\right]=\exp \left(2 \pi a^{t} \hat{x}\right) \boldsymbol{H}^{(n)}\left[\begin{array}{l}0 \\ 0\end{array}\right]$.

In the next article, we shall be concerned with an application to quantum mechanics. 
Notations.

$Z_{\geqq 0}=\{$ non-negative integer $\}$,

$Z_{\geqq 0}^{g}=\left\{j=\left(j_{1}, \cdots, j_{g}\right) \mid j_{i} \in Z_{\geqq 0}\right\}$,

$|j|=j_{1}+\cdots+j_{g}$,

$j \pm \varepsilon_{i}=\left(j_{1}, \cdots, j_{i-1}, j_{i} \pm 1, j_{i+1}, \cdots, j_{g}\right)$,

$\left(2 \pi n \sqrt{-1} x+\frac{\partial}{\partial \hat{x}}\right)^{j}=\left(2 \pi n \sqrt{-1} x_{1}+\frac{\partial}{\partial \hat{x}_{1}}\right)^{j_{1}} \cdots\left(2 \pi n \sqrt{-1} x_{g}+\frac{\partial}{\partial \hat{x}_{g}}\right)^{j_{g}}$

$\left(x+\ell+\frac{a}{n}\right)^{j}=\left(x_{1}+\ell_{1}+\frac{a_{1}}{n}\right)^{j_{1}} \cdots\left(x_{g}+\ell_{g}+\frac{a_{g}}{n}\right)^{j_{g}}$.

§1. Equivariant isomorphisms of $L^{2}\left(R^{g}\right)$ into $L^{2}\left(H_{2 g+1}(Z) \backslash H_{2 g+1}(R)\right)$

1.1. First we choose a complex symmetric $g \times g$ matrix $\tau=\tau^{\prime}+$ $\sqrt{-1} \tau^{\prime \prime}$ with positive definite imaginary part $\tau^{\prime \prime}$, and fix $\tau$ once for all. A system of complex coordinates is introduced,

$$
z=\hat{x}+x \tau, \quad \bar{z}=\hat{x}+x \bar{\tau} .
$$

Real and complex coordinates are related as follows

$$
\begin{gathered}
\frac{\partial}{\partial \hat{x}}=\frac{\partial}{\partial z}+\frac{\partial}{\partial \bar{z}}, \quad \frac{\partial}{\partial x}=\tau \frac{\partial}{\partial z}+\bar{\tau} \frac{\partial}{\partial z} \\
\tau \frac{\partial}{\partial \hat{x}}-\frac{\partial}{\partial x}=(\tau-\bar{\tau}) \frac{\partial}{\partial \bar{z}}=2 \sqrt{-1} \tau^{\prime \prime} \frac{\partial}{\partial z}
\end{gathered}
$$

Let us recollect the definition of theta functions. An entire function $f(z)$ in $z$ is called a theta function of level $n$ with respect to $\tau$, if it satisfies

$$
\begin{gathered}
f(z+\hat{b}+b \tau)=\exp \left(-\pi n \sqrt{-1}\left(b \tau^{t} b+2 z^{t} b\right)\right) f(z) \\
\left((\hat{b}, b) \in Z^{g} \times Z^{g}\right) .
\end{gathered}
$$

The space $\Theta_{0}^{(n)}$ of theta functions of level $n$ is a vector space of dimension $n^{g}$ with a basis consisting of theta series of level $n$

$$
\begin{gathered}
\vartheta^{(n)}\left[\begin{array}{c}
a / n \\
0
\end{array}\right](\tau \mid z)=\sum_{\ell \in Z^{g}} \exp \left(\pi n \sqrt{-1}\left(\left(\ell+\frac{a}{n}\right) \tau^{t}\left(\ell+\frac{a}{n}\right)+2 z^{t}\left(\ell+\frac{a}{n}\right)\right)\right) \\
\left(a \in Z^{g} / n Z^{g}\right) .
\end{gathered}
$$

A function $\varphi(u, z)$ in $2 g$ complex variables $(u, z)=\left(u_{1}, \cdots, u_{g}, z_{1}, \cdots, z_{g}\right)$ is called an auxiliary theta function of level $n$ (with respect to $\tau$ ), if it satisfies, 
i) $\varphi(u, z)$ is a polynomial in $z$ whose coefficient are entire functions in $u$.
ii) $\varphi(u+b, z+\hat{b}+b \tau)=\exp \left(-\pi n \sqrt{-1}\left(b \tau^{t} b+2 z^{t} b\right)\right) \varphi(u, z)$$$
\left((\hat{b}, b) \in \boldsymbol{Z}^{g} \times \boldsymbol{Z}^{g}\right) \text {. }
$$

In the previous article ${ }^{1)}$ the author proved that the space $\Theta^{(n)}$ of auxiliary theta functions of level $n$ has a basis consisting of auxiliary theta series of level $n$,

$$
\begin{aligned}
\vartheta_{\jmath}^{(n)}\left[\begin{array}{c}
a / n \\
0
\end{array}\right](\tau \mid u, z)= & \left(2 \pi n \sqrt{-1} u+\frac{\partial}{\partial z}\right)^{j} \vartheta^{(n)}\left[\begin{array}{c}
a / n \\
0
\end{array}\right](\tau \mid z) \\
= & \langle 2 \pi n \sqrt{-1})^{\prime, j !} \sum_{\ell \in Z^{g}}\left(u+\ell+\frac{a}{n}\right)^{j} \\
& \cdot \exp \left(\pi n \sqrt{-1}\left(\left(\ell+\frac{a}{n}\right) \tau^{t}\left(\ell+\frac{a}{n}\right)+2 z^{t}\left(\ell+\frac{a}{n}\right)\right)\right) \\
& \left(a \in Z^{g} / n Z^{g}, j \in Z_{\geq 0}^{g}\right)
\end{aligned}
$$

A mixed theta function of level $n$ (with respect to $\tau$ ) mean a real analytic function $\varphi(\hat{x}, x)$ in $(\hat{x}, x)$ such that

i) $\varphi(\hat{x}, x)$ is a polynomial in $x$ whose coefficients are entire function in complex variables $z=\hat{x}+x \tau$,

ii) $\varphi(\hat{x}+\hat{b}, x+b)=\exp \left(-\pi n \sqrt{-1}\left(b \tau^{t} b+2(\hat{x}+x \tau)^{t} b\right)\right) \varphi(\hat{x}, x)$

$$
\left((\hat{b}, b) \in Z^{g} \times Z^{g}\right) \text {. }
$$

It will be shown soon that the space $\Theta_{\text {mix }}^{(n)}$ of mixed theta functions of level $n$ (with respect to $\tau$ ) has a basis consisting of mixed theta series of level $n$,

$$
\begin{aligned}
& \vartheta_{j}^{(n)}\left[\begin{array}{c}
a / n \\
0
\end{array}\right](\tau \mid x, \hat{x}+x \tau) \\
& =(2 \pi n \sqrt{-1})^{|j|} \sum_{\ell \in Z^{g}}\left(x+\ell+\frac{a}{n}\right)^{j} \\
& \quad \cdot \exp \left(\pi n \sqrt{-1}\left(\left(\ell+\frac{g}{n}\right) \tau^{c}\left(\ell+\frac{a}{n}\right)+2\left(\hat{x}+x_{\tau}\right)^{t}\left(\ell+\frac{a}{n}\right)\right)\right) \\
& \quad\left(a \in Z^{g} / n Z^{g}, j \in Z_{\geq 0}^{g}, n \geq 1\right),
\end{aligned}
$$

which are the specializations of auxiliary theta series with respect to $(u, z) \mapsto(x, \hat{x}+x \tau)$.

1.2. Let us introduced a family of real analytic functions

1) See [3]. 


$$
\begin{aligned}
\phi_{j}^{(n)} & {\left[\begin{array}{c}
a / n \\
0
\end{array}\right]\left(\tau \mid x_{0}, \hat{x}, x\right) } \\
= & (2 \pi n \sqrt{-1})^{-|j|} \exp \left(\pi n \sqrt{-1}\left(x \tau^{t} x+2 \hat{x}^{t} x-2 x_{0}\right)\right) \vartheta_{j}^{(n)}\left[\begin{array}{c}
a / n \\
0
\end{array}\right](\tau \mid x, \hat{x}+x \tau) \\
= & \exp \left(-2 \pi n \sqrt{-1} x_{0}\right) \sum_{\ell \in Z^{g}}\left(x+\ell+\frac{a}{n}\right) \\
& \cdot \exp \left(\pi n \sqrt{-1}\left(\left(x+\ell+\frac{a}{n}\right) \tau^{t}\left(x+\ell+\frac{a}{n}\right)+2 \hat{x}^{t}\left(x+\ell+\frac{a}{n}\right)\right)\right) \\
& \left(a \in Z^{g} / n Z^{g}, j \in Z^{g}, n \geqq 1\right) .
\end{aligned}
$$

Proposition 1.1.

$$
\begin{gathered}
\left.\phi_{j}^{(n)}\left[\begin{array}{c}
a / n \\
0
\end{array}\right]\left(\tau \mid x_{0}, \hat{x}, x\right)=\exp (2 \pi \sqrt{-1}) a^{t} \hat{x}\right) \phi_{j}\left[\begin{array}{l}
0 \\
0
\end{array}\right]\left(\left(\tau \mid x_{0}, \hat{x}, x\right) \circ\left(0,0, \frac{a}{n}\right)\right), \\
\phi_{j}^{(n)}\left[\begin{array}{c}
a / n \\
0
\end{array}\right]\left(\tau \mid\left(b_{0}, \hat{b}, b\right) \circ\left(x_{0}, \hat{x}, x\right)\right)=\phi_{j}^{(n)}\left[\begin{array}{c}
a / n \\
0
\end{array}\right]\left(\tau \mid x_{0}, \hat{x}, x\right) \\
\left(\left(b_{0}, \hat{b} b\right) \in H_{2 g+1}(Z), a \in Z^{g} / n Z^{g}, j \in Z_{\geqq 0}^{g}, n \geqq 1\right) .
\end{gathered}
$$

Proof. (1.8) is an immediate consequence of the definition of $\phi_{j}^{(n)}\left[\begin{array}{c}a / n \\ 0\end{array}\right]\left(\tau \mid x_{0}, \hat{x}, x\right)$. For each $\left(b_{0}, \hat{b}, b\right)$ in $H_{2 g+1}(Z)$ we have

$$
\begin{aligned}
\phi_{j}^{(n)}\left[\begin{array}{c}
a / n \\
0
\end{array}\right]\left(\left(b_{0}, \hat{b}, b\right) \circ\left(x_{0}, \hat{x}, x\right)\right) & \\
= & \phi_{j}^{(n)}\left[\begin{array}{c}
a / n \\
0
\end{array}\right]\left(b_{0}+x_{0}+\hat{b}^{t} x, \hat{b}+\hat{x}, b+x\right) \\
= & \exp \left(2 \pi n \sqrt{-1}\left(b_{0}+x_{0}+\hat{b}^{t} x\right)\right) \sum_{\ell \in Z^{g}}\left(x+b+\ell+\frac{a}{n}\right)^{j} \\
& \cdot \exp \left(\pi n \sqrt { - 1 } \left(\left(x+b+\ell+\frac{a}{n}\right) \tau^{t}\left(x+b+\ell+\frac{a}{n}\right)\right.\right. \\
& \left.\left.+2(\hat{x}+\hat{b})^{t}\left(x+b+\ell+\frac{a}{n}\right)\right)\right) \\
= & \phi_{j}^{(n)}\left[\begin{array}{c}
a / n \\
0
\end{array}\right]\left(\tau \mid x_{0}, \hat{x}, x\right) .
\end{aligned}
$$

Proposition 1.1 means that $\phi_{j}^{(n)}\left[\begin{array}{c}a / n \\ 0\end{array}\right]\left(\tau \mid x_{0}, \hat{x}, x\right), \phi_{j}^{(n)}\left[\begin{array}{c}a / n \\ 0\end{array}\right]\left(\tau \mid \hat{x}_{0}, x\right)$ are real analytic functions on the quotient space $H_{2 g+1}(Z) \backslash H_{2 g+1}(R)$.

1.3. Lie algebra $\mathfrak{h}_{2 g+1}(\boldsymbol{R})$ of left invariant vector fields on $H_{2 g+1}(\boldsymbol{R})$ has a basis 


$$
D_{0}=-\frac{\partial}{\partial x_{0}}, \quad \hat{D}_{i}=-\frac{\partial}{\partial \hat{x}_{i}}, \quad D_{i}=\frac{\partial}{\partial x_{i}}+\hat{x}_{i} \frac{\partial}{\partial x_{\mathrm{v}}} \quad(1 \leqq i \leqq g)
$$

such that

$$
\begin{aligned}
& {\left[D_{0}, \hat{D}_{i}\right]=\left[D_{0}, D_{i}\right]=\left[D_{i}, D_{k}\right]=\left[\hat{D}_{i}, \hat{D}_{k}\right]=0} \\
& {\left[D_{i}, \hat{D}_{k}\right]= \begin{cases}D_{0} & (i=k) \\
0 & (i \neq k)\end{cases} }
\end{aligned}
$$

\section{THEOREM 1.1.}

$$
\begin{gathered}
D_{0} \phi_{j}^{(n)}\left[\begin{array}{c}
a / n \\
0
\end{array}\right]\left(\tau \mid x_{0}, \hat{x}, x\right)=2 \pi n \sqrt{-1} \phi_{j}^{(n)}\left[\begin{array}{c}
a / n \\
0
\end{array}\right]\left(\tau \mid x_{0}, \hat{x}, x\right), \\
\hat{D}_{i} \phi_{j}^{(n)}\left[\begin{array}{c}
a / n \\
0
\end{array}\right]\left(\tau \mid x_{0}, \hat{x}, x\right)=2 \pi n \sqrt{-1} \phi_{j+\varepsilon_{i}}^{(n)}\left[\begin{array}{c}
a / n \\
0
\end{array}\right]\left(\tau \mid x_{0}, \hat{x}, x\right), \\
D_{i} \phi_{j}^{(n)}\left[\begin{array}{c}
a / n \\
0
\end{array}\right]\left(\tau \mid x_{0}, \hat{x}, x\right)=j_{i} \phi_{j-\varepsilon_{1}}^{(n)}\left[\begin{array}{c}
a / n \\
0
\end{array}\right]\left(\tau \mid x_{0}, \hat{x}, x\right) \\
+2 \pi n \sqrt{-1} \sum_{p=1}^{g} \tau_{i p} \phi_{j+\varepsilon_{p}}^{(n)}\left[\begin{array}{c}
a / n \\
0
\end{array}\right]\left(\tau \mid x_{0}, \hat{x}, \hat{x}\right) \\
\left(1 \leqq i \leqq g, a \in Z^{g} / n Z^{g}, j \in Z_{\geqq 0}^{g}, n \geqq 1\right) .
\end{gathered}
$$

Proof. From the definition of $\phi_{j}^{(n)}\left[\begin{array}{c}a / n \\ 0\end{array}\right]\left(\tau \mid x_{0}, \hat{x}, x\right)$ it follows

$$
\begin{aligned}
D_{0} \phi_{j}^{(n)} & {\left[\begin{array}{c}
a / n \\
0
\end{array}\right]\left(\tau \mid x_{0}, \hat{x}, x\right) } \\
= & -\frac{\partial}{\partial x_{0}}\left\{(2 \pi n \sqrt{-1})^{-|j|}\right. \\
& \left.\cdot \exp \left(\pi n \sqrt{-1}\left(x \tau^{t} x+2 \hat{x}^{t} x-2 x_{0}\right)\right) \vartheta_{j}^{(n)}\left[\begin{array}{c}
a / n \\
0
\end{array}\right](\tau \mid x, \hat{x}+x \tau)\right\} \\
= & 2 \pi n \sqrt{-1} \phi_{j}^{(n)}\left[\begin{array}{c}
a / n \\
0
\end{array}\right]\left(\tau \mid x_{0}, \hat{x}, x\right),
\end{aligned}
$$

$$
\begin{aligned}
\hat{D}_{i} \phi_{j}^{(n)} & {\left[\begin{array}{c}
a / n \\
0
\end{array}\right]\left(\tau \mid x_{0}, \hat{x}, x\right) } \\
= & \frac{\partial}{\partial \hat{x}_{i}}\left\{\exp \left(-2 \pi n \sqrt{-1} x_{0}\right) \sum_{\ell \in Z^{g}}\left(x+\ell+\frac{a}{n}\right)^{j}\right. \\
& \left.\cdot \exp \left(\pi n \sqrt{-1}\left(\left(x+\ell+\frac{a}{n}\right) \tau^{t}\left(x+\ell+\frac{a}{n}\right)+2 \hat{x}^{t}\left(x+\ell+\frac{a}{n}\right)\right)\right)\right\} \\
= & 2 \pi n \sqrt{-1} \exp \left(-2 \pi n \sqrt{-1} x_{0}\right) \sum_{\ell}\left(x+\ell+\frac{a}{n}\right)^{j+\varepsilon_{i}}
\end{aligned}
$$




$$
\begin{aligned}
& \cdot \exp \left(\pi n \sqrt{-1}\left(\left(x+\ell+\frac{a}{n}\right) \tau^{t}\left(x+\ell+\frac{a}{n}\right)+2 \hat{x}^{t}\left(x+\ell+\frac{a}{n}\right)\right)\right) \\
&= 2 \pi n \sqrt{-1} \phi_{j+\varepsilon_{i}}^{(n)}\left[\begin{array}{c}
a / n \\
0
\end{array}\right]\left(\tau \mid x_{0}, \hat{x}, x\right) \\
& D_{i} \phi_{j}^{(n)}\left[\begin{array}{c}
a / n \\
0
\end{array}\right]\left(\tau \mid x_{0}, \hat{x}, x\right) \\
&=\left(\frac{\partial}{\partial x_{i}}+\hat{x}_{i} \frac{\partial}{\partial x_{0}}\right)\left\{\exp \left(-2 \pi n \sqrt{-1} x_{0}\right) \sum_{\ell \in Z_{g}}\left(x+\ell+\frac{a}{n}\right)^{j}\right. \\
&\left.\cdot \exp \left(\pi n \sqrt{-1}\left(\left(x+\ell+\frac{a}{n}\right) \tau^{t}\left(x+\ell+\frac{a}{n}\right)+2 \hat{x}^{t}\left(x+\ell+\frac{a}{n}\right)\right)\right)\right\} \\
&= \exp \left(-2 \pi n \sqrt{-1} x_{0}\right) \sum_{\ell \in Z^{g}}\left\{j_{i}\left(x+\ell+\frac{a}{n}\right)^{j-\varepsilon_{i}}\right. \\
&\left.+2 \pi n \sqrt{-1}\left(-\hat{x}_{i}+\sum_{p=1}^{g} \tau_{i p}\left(x_{p}+\ell_{p}+\frac{a_{p}}{n}\right)+\hat{x}_{i}\right)\left(x+\ell+\frac{a}{n}\right)^{j}\right\} \\
& \cdot \exp \left(\pi n \sqrt{-1}\left(\left(x+\ell+\frac{a}{n}\right) \tau^{t}\left(x+\ell+\frac{a}{n}\right)+2 \hat{x}^{t}\left(x+\ell+\frac{a}{n}\right)\right)\right) \\
&= j_{i} \phi_{j-\varepsilon_{i}}^{(n)}\left[\begin{array}{c}
a / n \\
0
\end{array}\right]\left(\tau \mid x_{0}, \hat{x}, x\right)+2 \pi n \sqrt{-1} \sum_{p=1}^{g} \phi_{j+\varepsilon_{p}}^{(n)}\left[\begin{array}{c}
a / n \\
0
\end{array}\right]\left(\tau \mid x_{0}, \hat{x}_{-}^{\prime}, x\right) .
\end{aligned}
$$

\section{Corollary 1.1.1.}

$$
\begin{gathered}
\left(D_{i}-\sum_{p=1}^{g} \tau_{i p} \hat{D}_{p}\right) \phi_{j}^{(n)}\left[\begin{array}{c}
a / n \\
0
\end{array}\right]\left(\tau \mid x_{0}, \hat{x}, x\right)=j_{i} \phi_{j-\varepsilon_{i}}^{(n)}\left[\begin{array}{c}
a / n \\
0
\end{array}\right]\left(\tau \mid x_{0}, \hat{x}, x\right), \\
\left(\hat{D}_{i} D_{i}-\sum_{p=1}^{g} \tau_{i p} \hat{D}_{i} \hat{D}_{p}\right) \phi_{j}^{(n)}\left[\begin{array}{c}
a / n \\
0
\end{array}\right]\left(\tau \mid x_{0}, \hat{x}, x\right) \\
=2 \pi n \sqrt{-1} j_{i} \phi_{j}^{(n)}\left[\begin{array}{c}
a / n \\
0
\end{array}\right](\tau \mid x, \hat{x}, x) . \\
\left(1 \leqq i \leqq g, a \in Z^{g} / n Z^{g}, j \in Z_{\geqq 0}^{g} . n \geqq 1\right)
\end{gathered}
$$

These are direct consequences of (1.11), (1.12).

CorollaRY. $\quad \vartheta_{j}^{(n)}\left[\begin{array}{c}a / n \\ 0\end{array}\right](\tau \mid x, \hat{x}+x \tau)\left(a \in Z^{g} / n Z^{g}, j \in Z_{\geqq 0}^{g}, n \geqq 1\right)$ are linearly independent.

Proof. For each $\hat{a} \in Z^{g} / n Z^{g}$ we have

$$
\vartheta_{j}^{(n)}\left[\begin{array}{c}
a / n \\
0
\end{array}\right]\left(\tau \mid x, \hat{x}+\frac{\hat{a}}{n}+x \tau\right)
$$




$$
\begin{aligned}
= & (2 \pi n \sqrt{-1})^{|j|} \sum_{\ell \in Z^{g}}\left(x+\ell+\frac{a}{n}\right)^{j} \\
& \cdot \exp \left(\pi n \sqrt{-1}\left(\left(\ell+\frac{a}{n}\right) \tau^{t}\left(\ell+\frac{a}{n}\right)+2\left(\hat{x}+\frac{\hat{a}}{n}\right)^{t}\left(\ell+\frac{a}{n}\right)\right)\right) \\
= & \exp \left(2 \pi \sqrt{-1} \frac{\hat{a}^{t} a}{n}\right) \vartheta_{j}^{(n)}\left[\begin{array}{c}
a / n \\
0
\end{array}\right](\tau \mid x, \hat{x}+x \tau) .
\end{aligned}
$$

Hence by virtue of (1.10), (1.13), (1.14) and the above relation we conclude the linearly independence of

$\phi_{j}^{(n)}\left[\begin{array}{c}a / n \\ 0\end{array}\right]\left(\tau \mid x_{0}, \hat{x}, x\right)$

$$
=(2 \pi n \sqrt{-1})^{-|j|} \exp \left(\pi n \sqrt{-1}\left(x \tau^{t} x+2 \hat{x}^{t} x-2 x_{0}\right)\right) \vartheta_{j}^{(n)}\left[\begin{array}{c}
a / n \\
0
\end{array}\right](\tau \mid x, \hat{x}+x \tau) .
$$

\section{Denote}

$$
\begin{aligned}
\boldsymbol{H}_{\tau}^{(n)}\left[\begin{array}{c}
a / n \\
0
\end{array}\right]= & \text { the completion of the vector space } \\
& \text { spanned by } \phi_{j}^{(n)}\left[\begin{array}{c}
a / n \\
0
\end{array}\right]\left(\tau \mid x_{0}, \hat{x}, x\right) \quad\left(j \in Z_{\geqq 0}^{g}\right) \\
\overline{\boldsymbol{H}_{\tau}^{(n)}\left[\begin{array}{c}
a / n \\
0
\end{array}\right]} & =\text { the complex conjugate of } \boldsymbol{H}_{\tau}^{(n)}\left[\begin{array}{c}
a / n \\
0
\end{array}\right] .
\end{aligned}
$$

THEOREM 1.2. $\boldsymbol{H}_{\tau}^{(n)}\left[\begin{array}{c}a / n \\ 0\end{array}\right], \overline{\boldsymbol{H}_{\tau}^{(n)}\left[\begin{array}{c}a / n \\ 0\end{array}\right]}$ are irreducible invariant subspace of $L^{2}\left(H_{2 g+1}(Z) \backslash H_{2 g+1}(R)\right)$ with respect to the unitary representation $\rho$ :

$$
\rho\left(y_{0}, \hat{y}, y\right) \phi\left(x_{0}, \hat{x}, x\right)=\phi\left(\left(x_{0}, \hat{x}, x\right) \circ\left(y_{0}, \hat{y}, y\right)\right)
$$

such that

$$
\begin{aligned}
& H^{(n)}\left[\begin{array}{c}
a / n \\
0
\end{array}\right]=\exp \left(2 \pi a^{t} \hat{x}\right) H^{(n)}\left[\begin{array}{l}
0 \\
0
\end{array}\right], \\
& \rho\left(y_{0}, 0,0\right) \phi=\exp \left(-2 \pi n \sqrt{-1} y_{0}\right) \phi \quad\left(\phi \in \boldsymbol{H}_{\tau}^{(n)}\left[\begin{array}{c}
a / n \\
0
\end{array}\right]\right), \\
& \rho\left(y_{0} 0,0\right) \bar{\phi}=\exp \left(2 \pi n \sqrt{-1} y_{0}\right) \bar{\phi} \quad\left(\bar{\phi} \overline{\left.\boldsymbol{H}_{\tau}^{(n)}\left[\begin{array}{c}
a / n \\
0
\end{array}\right]\right) .}\right.
\end{aligned}
$$

Proof. Theorem 1.1 states that the Lie algebra representation $d \rho$ of $\mathfrak{h}_{2 g}(\boldsymbol{R})$ |preserves $\boldsymbol{H}_{\tau}^{(n)}\left[\begin{array}{c}a / n \\ 0\end{array}\right], \overline{\boldsymbol{H}_{\tau}^{(n)}\left[\begin{array}{c}a / n \\ 0\end{array}\right]}$ and 


$$
\begin{array}{ll}
d \rho\left(D_{0}\right) \phi=-\frac{\partial}{\partial x_{0}} \phi=2 \pi n \sqrt{-1} \phi & \left(\phi \in \boldsymbol{H}_{\tau}^{(n)}\left[\begin{array}{c}
a / n \\
0
\end{array}\right]\right), \\
d \rho\left(D_{0}\right) \bar{\phi}=\frac{\partial}{\partial x_{0}} \phi=-2 \pi n \sqrt{-1} \phi & \left(\bar{\phi} \in \boldsymbol{H}_{\tau}^{(n)}\left[\begin{array}{c}
a / n \\
0
\end{array}\right]\right) .
\end{array}
$$

Since $\phi_{j}^{(n)}\left[\begin{array}{c}a / n \\ 0\end{array}\right]\left(\tau \mid x_{0}, \hat{x}, x\right)=\exp \left(2 \pi \sqrt{-1} a^{t} \hat{x}\right) \phi_{j}^{(n)}\left[\begin{array}{l}0 \\ 0\end{array}\right]\left(\tau \mid\left(x_{0}, \hat{x}, x\right) \circ(0,0, a / n)\right)$, we complete the proof of Theorem 1.2.

1.4. Let $L^{2}\left(\boldsymbol{R}^{g}, \mu_{\tau^{\prime \prime}}^{(n)}\right)$ be the $L^{2}$-space of $\boldsymbol{R}^{g}$ with respect to the measure

$$
\pi_{\tau^{\prime \prime}}^{(n)}(d \xi)=\exp \left(-2 \pi n \xi \tau^{\prime \prime} \xi\right) d \xi,
$$

where $n \geqq 1$.

LEMMA 1.1. The transformation

$$
f(\xi) \longmapsto \exp \left(-\pi n \sqrt{-1} \xi \tau^{t} \xi\right) f(\xi)
$$

is an isomorphism of Hilbert space $L^{2}\left(\boldsymbol{R}^{g}, \mu_{\tau^{\prime \prime}}^{(n)}\right)$ onto $L^{2}\left(\boldsymbol{R}^{g}\right)$.

Proof. Since $\tau-\bar{\tau}=2 \sqrt{-1} \tau^{\prime \prime}$, we have

$$
\begin{gathered}
\int_{R^{g}} \overline{\exp \left(-\pi n \sqrt{-1} \xi \tau^{t} \xi\right)} \exp \left(\pi n \sqrt{-1} \xi \tau^{t} \xi\right) f_{2}(\xi) d \xi \\
=\int_{R^{g}} \exp \left(-2 \pi n \xi \tau^{\prime \prime} \xi\right) \overline{f_{1}(\xi)} f_{2}(\xi) d \xi .
\end{gathered}
$$

Hence the transformations (1.15) is an isomorphism of $L^{2}\left(\boldsymbol{R}, \mu_{\tau^{\prime \prime}}^{(n)}\right)$ onto $L^{2}\left(R^{g}\right)$.

Since the set of monomials $\left\{\xi^{j} \mid j \in Z_{\geqq 0}^{g}\right\}$ is a basis of $L^{2}\left(R^{g}, \mu_{\tau^{\prime \prime}}^{(n)}\right)$, the set of functions $\left\{\exp \left(\pi n \sqrt{-1} \xi \tau^{t} \xi\right) \xi^{j} \mid j \in Z_{\geqq 0}^{g}\right\}$ is a basis of $L^{2}\left(\boldsymbol{R}^{g}\right)$.

LEMMA 1.2.

$$
\begin{gathered}
\int_{H_{2 g+1}(\boldsymbol{Z}) \backslash H_{2 g}(\boldsymbol{R})} \phi_{j}^{(n)}\left[\begin{array}{c}
a / n \\
0
\end{array}\right]\left(\tau \mid x_{0}, \hat{x}, x\right) \phi_{k}^{(m)}\left[\begin{array}{c}
c / m \\
0
\end{array}\right]\left(\tau \mid x_{0}, \hat{x}, x\right) d x_{0} d \hat{x} d x \\
= \begin{cases}\int_{R^{g}} y^{j+k} \exp \left(-2 \pi n y \tau^{\prime \prime t} y\right) d y & (n=m, a \equiv C \bmod n) \\
0 & \text { otherwise. }\end{cases}
\end{gathered}
$$

Proof. Let us first integrate on fibers

$$
\begin{aligned}
\left\langle\phi_{j}^{(n)}\right. & {\left.\left[\begin{array}{c}
a / n \\
0
\end{array}\right]\left(\tau \mid x_{0}, \hat{x}, x\right), \phi_{k}^{(m)}\left[\begin{array}{c}
c / m \\
0
\end{array}\right]\left(\tau \mid x_{0}, \hat{x}, x\right)\right\rangle } \\
& =\int_{H_{2 g+1}(Z) \backslash H_{g+1}(R)} \phi_{j}^{(n)}\left[\begin{array}{c}
a / n \\
0
\end{array}\right]\left(\tau \mid x_{0}, \hat{x}, x\right) \phi_{k}^{(m)}\left[\begin{array}{c}
c / m \\
0
\end{array}\right](\tau / x, \hat{x}, x) d x_{0} d \hat{x} d x
\end{aligned}
$$




$$
\begin{aligned}
= & \sum_{\ell, \ell^{\prime} \in Z^{g}} \int_{Z^{g} \backslash \boldsymbol{R}^{g}} \int_{Z^{g} \backslash \boldsymbol{R}^{g}}\left\{\int_{Z^{g} \backslash \boldsymbol{R}} \exp \left(-2 \pi \sqrt{-1}(-n+m) x_{0}\right)\right. \\
& \cdot d x_{0}\left(x+\ell+\frac{a}{n}\right)^{j}\left(x+\ell^{\prime}+\frac{c}{m}\right)^{k} \\
& \cdot \exp \left(\pi \sqrt{-1}\left(\ell+\frac{a}{n}\right) \bar{\tau}^{t}\left(\ell+\frac{a}{n}\right)+m\left(\ell^{\prime}+\frac{c}{m}\right) \tau^{t}\left(\ell^{\prime}+\frac{c}{m !}\right)\right) \\
& \left.\cdot \exp 2 \pi \sqrt{-1}\left(\hat{x}\left(-n^{t}\left(\ell+\frac{a}{n}\right)+m^{t}\left(\ell^{\prime}+\frac{c}{m}\right)\right)\right)\right\} d \hat{x} d x
\end{aligned}
$$

This means that

$$
\begin{aligned}
& \left\langle\phi_{j}^{(n)}\left[\begin{array}{c}
a / n \\
0
\end{array}\right]\left(\tau \mid x_{0}, \hat{x}, x\right), \phi_{k}^{(m)}\left[\begin{array}{c}
c / m \\
0
\end{array}\right]\left(\tau \mid x_{0}, \hat{x}, x\right)\right\rangle=0 \quad(n \neq m), \\
& \left\langle\phi_{j}^{(n)}\left[\begin{array}{c}
a / n \\
0
\end{array}\right]\left(\tau \mid x_{0}, \hat{x}, x\right), \phi_{k}^{(n)}\left[\begin{array}{c}
c / n \\
0
\end{array}\right]\left(\tau \mid x_{0}, \hat{x}, x\right)\right\rangle=0 \quad(a \neq c \bmod n) \\
& \left\langle\phi_{j}^{(n)}\left[\begin{array}{c}
a / n \\
0
\end{array}\right]\left(\tau \mid x_{0}, \hat{x}, x\right), \phi_{k}^{(n)}\left[\begin{array}{c}
a / n \\
0
\end{array}\right]\left(\tau \mid x_{0}, \hat{x}, x\right)\right\rangle \\
& \quad=\sum_{\ell \in Z^{g}} \int_{Z^{g} \backslash Z^{g}}\left(x+\ell+\frac{a}{n}\right)^{j+k} \exp -2 \pi n\left(x+\ell+\frac{a}{n}\right) \tau^{\prime \prime}\left(x+\ell+\frac{a}{n}\right) d x \\
& \quad=\int_{R^{g}} y^{j+k} \exp \left(-2 \pi n y \tau^{\prime \prime t} y\right) d y .
\end{aligned}
$$

LEMMA 1.3. The transformations of $L^{2}\left(\boldsymbol{R}^{g}, \mu_{\tau^{\prime \prime}}^{(n)}\right)$ onto $\boldsymbol{H}^{(n)}\left[\begin{array}{c}a / n \\ 0\end{array}\right]$ given by

$$
\xi_{j} \longmapsto \phi_{j}^{(n)}\left[\begin{array}{c}
a / n \\
0
\end{array}\right]\left(\tau \mid x_{0} \hat{x}, x\right) \quad\left(j \in Z_{\geqq 0}^{g}\right)
$$

is an isomorphism of Hilbert spaces.

This is an immediate consequence of Lemma 1.2.

We define unitary action of $H_{2 g+1}(R)$ on $L^{2}(R)$ by

$$
\chi_{n}\left(x_{0}, \hat{x}, x\right) f(\xi)=\exp \left(-2 \pi n \sqrt{-1}\left(x_{0}+x^{t} \xi\right)\right) f(\xi+\hat{x}) .
$$

THEOREM 1.3. Let $\phi_{\tau}^{(n)}\left[\begin{array}{c}a / n \\ 0\end{array}\right]$ be the transformation of $L^{2}\left(\boldsymbol{R}^{g}\right)$ onto $\boldsymbol{H}_{\tau}^{(n)}\left[\begin{array}{c}a / n \\ 0\end{array}\right]$ given by

$$
\begin{array}{r}
\phi_{\tau}^{(n)}\left[\begin{array}{c}
a / n \\
0
\end{array}\right]\left(\exp \left(\pi n \sqrt{-1} \xi \tau^{t} \xi\right) \xi^{j}\right)\left(x_{0}, \hat{x},{ }^{\top} x\right) \\
\quad=\phi_{j}^{(n)}\left[\begin{array}{c}
a / n \\
0
\end{array}\right]\left(\tau \mid x_{0}, \hat{x}, x\right) \quad\left(j \in Z_{\geqq 0}^{g}\right) .
\end{array}
$$


Then $\phi_{\tau}^{(n)}\left[\begin{array}{c}a / n \\ 0\end{array}\right]$ is an isomorphism of Hilbert space $L^{2}\left(\boldsymbol{R}^{g}\right)$ to Hilbert space $\boldsymbol{H}_{\tau}^{(n)}\left[\begin{array}{c}a / n \\ 0\end{array}\right]$ such that

$$
\begin{gathered}
\rho\left(y_{0}, \hat{y}, y\right) \circ \phi_{\tau}^{(n)}\left[\begin{array}{c}
a / n \\
0
\end{array}\right]=\phi_{\tau}^{(n)}\left[\begin{array}{c}
a / n \\
0
\end{array}\right] \circ \chi_{n}\left(y_{0}, \hat{y}, y\right) \\
\left(\left(y_{0}, \hat{y}, y\right) \in H_{2 g+1}(R)\right), \\
\phi_{\tau}^{(n)}\left[\begin{array}{c}
a / n \\
0
\end{array}\right]=\exp \left(2 \pi \sqrt{-1} a^{t} \hat{x}\right) \rho\left(0,0, \frac{a}{n}\right) \phi_{\tau}^{(n)}\left[\begin{array}{l}
0 \\
0
\end{array}\right] .
\end{gathered}
$$

Proof. By virtue of Lemmas 1.2 and $1.3 \phi_{\tau}^{(n)}\left[\begin{array}{c}a / n \\ 0\end{array}\right]$ is an isomorphism of Hilbert spaces. Let us prove the equivariance of $\phi_{\tau}^{(n)}\left[\begin{array}{c}a / n \\ 0\end{array}\right]$. From the action

$$
\begin{aligned}
& \chi_{n}\left(x_{0}, \hat{x}, x\right)\left(\exp \left(\pi n \sqrt{-1} \xi \tau^{t} \xi\right) \xi^{j}\right) \\
& \quad=\exp \left(-2 \pi n \sqrt{-1}\left(x_{0}+x^{t} \xi\right)\right) \exp \left(\pi n \sqrt{-1}(\xi+\hat{x}) \tau^{t}(\xi+\hat{x})\right)(\xi+\hat{x})^{j},
\end{aligned}
$$

we have

$$
\begin{aligned}
& d \chi_{n}\left(D_{0}\right)\left(\exp \left(\pi n \sqrt{-1} \xi \tau^{t}\right) \xi^{j}\right) \\
& \quad=-\left.\frac{\partial}{\partial x_{0}}\right|_{x_{0}=0}\left(\chi_{n}\left(x_{0}, \hat{x}, x\right)-1\right)\left(\exp \left(\pi n \sqrt{-1} \xi \tau^{t} \xi\right) \xi^{j}\right) \\
& \quad=-2 \pi n \sqrt{-1} \exp \left(\pi n \sqrt{-1} \xi \tau^{t} \xi\right) \xi^{j} \\
& d \chi_{n}\left(D_{i}\right)\left(\exp \left(\pi n \sqrt{-1} \xi \tau^{t} \xi\right) \xi^{j}\right) \\
& \quad=-2 \pi n \sqrt{-1} \xi_{i} \exp \left(\pi n \sqrt{-1} \xi \tau^{t} \xi\right) \xi^{j} \\
& \quad=-2 \pi n \sqrt{-1} \exp \left(\pi n \sqrt{-1} \xi \tau^{t} \xi\right) \xi^{j+\varepsilon_{i}}, \\
& d \chi_{n}\left(\hat{D}_{i}\right)\left(\exp \left(\pi n \sqrt{-1} \xi \tau^{t} \xi\right) \xi^{j}\right) \\
& \quad=\exp \left(\pi n \sqrt{-1} \xi \tau^{t} \xi\right)\left(2 \pi n \sqrt{-1}\left(\sum_{p=1}^{g} \tau_{i p} \xi_{p}\right) \xi^{j}+j_{i} \xi^{j-\varepsilon_{i}}\right) \\
& \quad=2 \pi \sqrt{-1} \sum_{p=1}^{g} \tau_{i p} \exp \left(\pi n \sqrt{-1} \xi \tau^{t} \xi\right) \xi^{j+\varepsilon} p+j_{i} \exp \left(\pi n \sqrt{-1} \xi \tau^{t} \xi\right) \xi^{j-\varepsilon_{i}} .
\end{aligned}
$$

Hence by virtue of (1.10), (1.11), (1.12) we have

$$
\begin{aligned}
\left(\phi_{\tau}^{(n)}\right. & {\left.\left[\begin{array}{c}
a / n \\
0
\end{array}\right] \circ d \chi_{n}\left(D_{0}\right)\right)\left(\xi^{j} \exp \left(\pi n \sqrt{-1} \xi \tau^{t} \xi\right)\right)\left(x_{0}, \hat{x}, x\right) } \\
& =2 \pi n \sqrt{-1} \phi_{\tau}^{(n)}\left[\begin{array}{c}
a / n \\
0
\end{array}\right](\tau \mid x, \hat{x}, x) \\
& =d \rho\left(D_{0}\right) \phi_{\tau}^{(n)}\left[\begin{array}{c}
a / n \\
0
\end{array}\right]\left(\xi^{j} \exp \left(\pi n \sqrt{-1} \xi \tau^{t} \xi\right)\right)\left(x_{0}, \hat{x}, x\right)
\end{aligned}
$$




$$
\begin{aligned}
\left(\phi_{\tau}^{(n)}\left[\begin{array}{c}
a / n \\
0
\end{array}\right] \circ d \chi_{n}\left(-D_{i}\right)\right)\left(\xi^{j} \exp \left(\pi n \sqrt{-1} \xi \tau^{t} \xi\right)\right)\left(x_{0}, \hat{x}, x\right) \\
=2 \pi n \sqrt{-1} \phi_{\tau}^{(n)}\left[\begin{array}{c}
a / n \\
0
\end{array}\right]\left(\xi^{j+\varepsilon_{i}} \exp \left(\pi n \sqrt{-1} \xi \tau^{t} \xi\right)\right)\left(x_{0}, \hat{x}, x\right) \\
=2 \pi n \sqrt{-1} \phi_{j+\varepsilon_{i}}^{(n)}\left[\begin{array}{c}
a / n \\
0
\end{array}\right]\left(\tau \mid x_{0}, \hat{x}, x\right)=d \rho\left(\hat{D}_{i}\right) \phi_{j}^{(n)}\left[\begin{array}{c}
a / n \\
0
\end{array}\right]\left(\tau \mid x_{0}, \hat{x}, x\right) \\
=\left(d \rho\left(\hat{D}_{i}\right) \circ \phi_{\tau}^{(n)}\left[\begin{array}{c}
a / n \\
0
\end{array}\right]\right)\left(\xi^{j} \exp \left(\pi n \sqrt{-1} \xi \tau^{t} \xi\right)\right)\left(x_{0}, \hat{x}, x\right), \\
\left(\phi_{\tau}^{(n)}\left[\begin{array}{c}
a / n \\
0
\end{array}\right] \circ d \chi_{n}\left(\hat{D}_{i}\right)\right)\left(\xi_{j} \exp \left(\pi n \sqrt{-1} \xi \tau^{t} \xi\right)\right)\left(x_{0}, \hat{x}, x\right) \\
=j_{i} \phi_{\tau}^{(n)}\left[\begin{array}{c}
a / n \\
0
\end{array}\right]\left(\xi^{j-\varepsilon_{i}} \exp \left(\pi n \sqrt{-1} \xi \tau^{t} \xi\right)\right)\left(x_{0}, \hat{x}, x\right)+2 \pi n \sqrt{-1} \sum_{p=1}^{g} \tau_{i p} \phi_{\tau}^{(n)}\left[\begin{array}{c}
a / n \\
0
\end{array}\right] \\
\quad \cdot\left(\xi^{j+\varepsilon} p \exp \left(\pi n \sqrt{-1} \xi \tau^{t} \xi\right)\right)\left(x_{0}, \hat{x}, x\right) \\
=j_{i} \phi_{j-\varepsilon_{i}}^{(n)}\left[\begin{array}{c}
a / n \\
0
\end{array}\right]\left(\tau \mid x_{0}, \hat{x}, x\right)+2 \pi n \sqrt{-1} \sum_{p=1}^{g} \tau_{i p} \phi_{j+\varepsilon_{p}}^{(n)}\left[\begin{array}{c}
a / n \\
0
\end{array}\right]\left(\tau \mid x_{0}, \hat{x}, x\right) \\
=d \rho\left(D_{i}\right) \phi_{j}^{(n)}\left[\begin{array}{c}
a / n \\
0
\end{array}\right]\left(\tau \mid x_{0}, \hat{x}, x\right) \\
=d \rho\left(D_{i}\right) \circ \phi_{\tau}^{(n)}\left[\begin{array}{c}
a / n \\
0
\end{array}\right]\left(\xi^{j} \exp \left(\pi n \sqrt{-1} \xi \tau^{t} \xi\right)\right)\left(x_{0}, \hat{x}, x\right) .
\end{aligned}
$$

This means

$$
d \rho\left(\begin{array}{cc}
0 & 1 \\
-1 & 0
\end{array}\right) \circ \phi_{\tau}^{(n)}\left[\begin{array}{c}
a / n \\
0
\end{array}\right]=\phi_{\tau}^{(n)}\left[\begin{array}{c}
a / n \\
0
\end{array}\right] \circ d \chi_{n}
$$

and thus

$$
\left.\rho^{(-1} \begin{array}{cc}
1 & 1
\end{array}\right) \circ \phi_{\tau}^{(n)}\left[\begin{array}{c}
a / n \\
0
\end{array}\right]=\phi_{\tau}^{(n)}\left[\begin{array}{c}
a / n \\
0
\end{array}\right] \circ \chi_{n},
$$

where

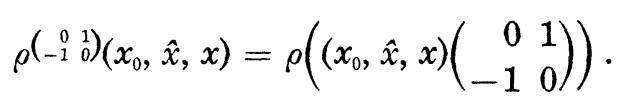

\section{$\S 2$. Decomposition of unitary representation $\rho$}

2.1. An irreducible unitary representation $\rho_{\lambda}$ of $H_{2 g+1}(\boldsymbol{R})$ is characterized by a real number $\lambda$ such that

$$
\rho_{\lambda}\left(y_{0}, 0,0\right) \phi=\exp \left(-2 \pi \lambda \sqrt{-1} y_{0}\right) \phi
$$

provided $\lambda \neq 0$. If $\lambda=0$, then it is characterized by a pair $(\hat{k}, k) \in \boldsymbol{R}^{g} \times \boldsymbol{R}^{g}$ of vectors as follows 


$$
\rho_{\hat{k}, k}\left(y_{0}, \hat{y}, y\right) \phi=\exp \left(2 \pi \sqrt{-1}\left(\hat{k}^{t} \hat{y}+k^{t} y\right) \phi .\right.
$$

For each integer $b_{0}$ and $\phi\left(x_{0}, \hat{x}, x\right)$ in $L^{2}\left(H_{2 g+1}(Z) \backslash H_{2 g+1}(R)\right)$

$$
\begin{aligned}
\phi\left(x_{0}, \hat{x}, x\right) & =\phi\left(\left(b_{0}, 0,0\right) \circ\left(x_{0}, \hat{x}, x\right)\right)=\phi\left(\left(x_{0}, \hat{x}, x\right) \circ\left(b_{0}, 0,0\right)\right) \\
& =\rho_{\lambda}\left(b_{0}, 0,0\right) \phi\left(x_{0}, \hat{x}, x\right)
\end{aligned}
$$

hence for every irreducible factor $\rho_{\lambda}$ of $\rho, \lambda$ must be an integer.

LEMMA 2.1. Let $\phi\left(x_{0}, \hat{x}, x\right)$ be a real analytic function on $H_{2 g+1}(Z) \backslash$ $H_{2 g+1}(R)$ such that

i) $\exp \left(2 \pi n \sqrt{-1} x_{0}\right) \phi\left(x_{0}, \hat{x}, x\right)$ is independent on $x_{0}$,

ii) $\left(D_{i}-\sum_{p=1}^{g} \tau_{i p} \hat{D}_{p}\right) \phi\left(x_{0}, \hat{x}, x\right)=0 \quad(1 \leqq i \leqq g)$.

Then

$$
\psi(\hat{x}, x)=\exp \left(-\pi n \sqrt{-1}\left(x \tau^{t} x+2 \hat{x}^{t} x-2 x\right)\right) \phi\left(x_{0}, \hat{x}, x\right)
$$

is a theta function of level $n$ in $z=\hat{x}+x \tau$ with respect to $\tau$.

Proof. For each $\left(b_{0}, \hat{b}, b\right)$ in $H_{2 g+}(Z)$ we have

$$
\begin{aligned}
\exp (- & \left.\pi n \sqrt{-1}\left((x+b) \tau^{t}(x+b)+2(\hat{x}+\hat{b})^{t}(x+b)-2 b_{0}-2 x-2 \hat{b}^{t} x\right)\right) \\
& \cdot \phi\left(\left(b_{0}, \hat{b}, b\right) \circ\left(x_{0}, \hat{x}, x\right)\right) \\
= & \exp \left(-\pi n \sqrt{-1}\left(b \tau^{t} b+2(\hat{x}+x \tau)^{t} b\right)\right) \\
& \cdot \exp \left(-\pi n \sqrt{-1}\left(x \tau^{t} x+2 \hat{x}^{t} x-2 x_{0}\right)\right) \phi\left(x_{0}, \hat{x}, x\right) .
\end{aligned}
$$

Hence we have the difference relation:

$$
\psi(\hat{x}+\hat{b}, x+b)=\exp \left(-\pi n \sqrt{-1}\left(b \tau^{t} b+2(\hat{x}+x \tau)^{t} b\right) \psi(\hat{x}, x) .\right.
$$

From the relation

$$
\tau \frac{\partial}{\partial \hat{x}}-\frac{\partial}{\partial x}=(\tau-\bar{\tau}) \frac{\partial}{\partial \bar{z}},
$$

in order to prove $\left(\partial / \partial \bar{z}_{i}\right) \psi(\hat{x}, x)=0(1 \leqq i \leqq g)$, it is sufficient to show

$$
\begin{gathered}
\left(\tau \frac{\partial}{\partial \hat{x}}-\frac{\partial}{\partial x}\right) \psi(\hat{x}, x)=0 . \\
\left(\sum_{p=1}^{g} \tau_{i p} \frac{\partial}{\partial \hat{x}_{p}}-\frac{\partial}{\partial x_{i}}\right) \psi(\hat{x}, x)=\left(\sum_{p=1}^{g} \tau_{i p} \frac{\partial}{\partial \hat{x}_{p}}-\frac{\partial}{\partial x_{i}}-\hat{x}_{i} \frac{\partial}{\partial x_{0}}\right) \psi(\hat{x}, x) \\
=\left(\sum_{p=1}^{g} \tau_{i p} \frac{\partial}{\partial \hat{x}_{p}}-\frac{\partial}{\partial x_{i}}-\hat{x}_{i} \frac{\partial}{\partial x_{0}}\right) \\
\cdot\left(\exp \left(-\pi n \sqrt{-1}\left(x \tau^{t} x+2 \hat{x}^{t} x-2 x_{0}\right)\right)\right) \phi\left(x_{0}, \hat{x}, x\right)
\end{gathered}
$$




$$
\begin{aligned}
& +\exp \left(-\pi n \sqrt{-1}\left(x \tau^{t} x+2 \hat{x}^{t} x-2 x_{0}\right)\right)\left(\sum_{p=1}^{g} \tau_{i p} \hat{D}_{p}-D_{i}\right) \phi\left(x_{0}, \hat{x}, x\right) \\
& =0 .
\end{aligned}
$$

Hence $\psi(\hat{x}, x)$ is an entire function in $z=\hat{x}+x \tau$ satisfying the difference equation for theta function of level $n$, and thus $\psi(\hat{x}, x)$ must be a theta function of level $n$.

THEOREM 2.1. Let $\boldsymbol{H}^{(n)}\left[\begin{array}{c}a / n \\ 0\end{array}\right]$ be the completion of the vector space

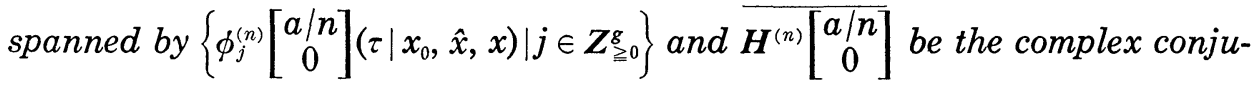

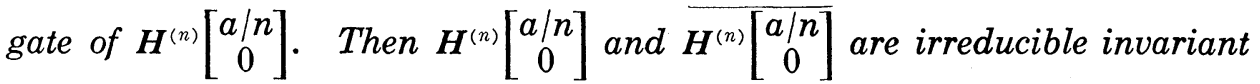
subspaces of $L^{2}\left(H_{2 g+1}(Z) \backslash H_{2 g+1}(R)\right)$ such that

$$
\begin{array}{r}
\rho\left(y_{0}, 0,0\right) \phi\left(x_{0}, \hat{x}, x\right)=\exp \left(-2 \pi n \sqrt{-1} y_{0}\right) \phi\left(x_{0}, \hat{x}, x\right) \\
\rho\left(y_{0}, 0,0\right) \overline{\phi\left(x_{0}, \hat{x}, x\right)}=\exp \left(-2 \pi n \sqrt{-1} y_{0}\right) \overline{\phi\left(x_{0}, \hat{x}, x\right)} \\
\left(\phi \in \boldsymbol{H}^{(n)}\left[\begin{array}{c}
a / n \\
0
\end{array}\right]\right),
\end{array}
$$

and the decomposition of $\rho$ is given by

$$
\begin{aligned}
& L^{2}\left(H_{2 g^{+1}}(Z) \backslash H_{2 g^{+1}}(R)\right)
\end{aligned}
$$

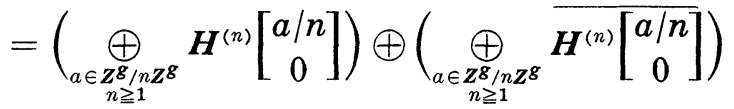

$$
\begin{aligned}
& \left(\underset{(\hat{k}, k) \in \boldsymbol{Z}^{\boldsymbol{g} \times \boldsymbol{Z}^{\boldsymbol{g}}}}{\bigoplus^{\prime}} \boldsymbol{C} \exp \left(2 \pi \sqrt{-1}\left(\hat{k}^{t} \hat{x}+k^{t} x\right)\right)\right) .
\end{aligned}
$$

The invariant subspaces

$$
H^{(n)}\left[\begin{array}{c}
a / n \\
0
\end{array}\right]=\exp \left(2 \pi a^{t} \hat{x}\right) \boldsymbol{H}^{(n)}\left[\begin{array}{l}
0 \\
0
\end{array}\right], \overline{\boldsymbol{H}^{(n)}\left[\begin{array}{c}
a / n \\
0
\end{array}\right]}=\exp \left(-2 \pi a^{t} \hat{x}\right) \overline{\boldsymbol{H}^{(n)}\left[\begin{array}{l}
0 \\
0
\end{array}\right]}
$$

are independent of the choice of $\tau$.

Proof. Since the space $A$ of real an analytic functions on $H_{2 g+1}(Z) \backslash$ $H_{2 g+1}(R)$ is dense in $L^{2}\left(H_{2 g+1}(Z) \backslash H_{2 g+1}(R)\right)$ and $A$ is invariant for $D_{0}, \hat{D}_{i}, D_{i}$ $(1 \leqq i \leqq g)$, i.e. for the action of $H_{2 g+1}(R)$, it is sufficient to decompose $A$. Let $W$ be an irreducible invariant subspace of $A$ such that $\rho\left(y_{0}, 0,0\right) \phi\left(x_{0}, \hat{x}, x\right)$ $=\exp \left(-2 \pi n \sqrt{-1} y_{0}\right) \phi\left(x_{0}, \hat{x}, x\right)\left(y_{0} \in R, \phi \in \bar{W}\right)$. If $n=0$, then there exists $(\hat{k}, k) \in \boldsymbol{Z}^{g} \times \boldsymbol{Z}^{g}$ such that

$$
W=C \exp \left(2 \pi \sqrt{-1}\left(\hat{k}^{t} \hat{x}+k^{t} x\right)\right) .
$$


If $n$ is negative, we replace $W$ by $\bar{W}$. So we may assume that $n$ is a positive integer. Let $\nu$ be a $\mathfrak{h}_{2 g+1}(\boldsymbol{R})$-module isomorphism of $\boldsymbol{H}^{(n)}\left[\begin{array}{c}a / n \\ 0\end{array}\right] \cap \boldsymbol{A}$ onto $\bar{W}$, i.e., a linear isomorphism satisfying

$$
D_{0} \circ \nu=\nu \circ D_{0}, \quad \hat{D}_{i} \circ \nu=\nu \circ \hat{D}_{i}, \quad D_{i} \circ \nu=\nu \circ D_{i} \quad(1 \leqq i \leqq g) .
$$

Since $\boldsymbol{H}^{(n)}\left[\begin{array}{c}a / n \\ 0\end{array}\right] \cap \boldsymbol{A}$ contains the element $\phi_{0}^{(n)}$ satisfying

$$
\left(D_{i}-\sum_{d=1}^{g} \tau_{i p} \hat{D}_{p}\right) \phi_{0}^{(n)}\left[\begin{array}{c}
a / n \\
0
\end{array}\right]\left(\tau \mid x_{0}, \hat{x}, x\right)=0 \quad(1 \leqq i \leqq g) .
$$

There exists an element $\phi_{0}\left(x_{0}, \hat{x}, x\right)$ in $\bar{W}$ such that

$$
\left(D_{i}-\sum_{p=1}^{g} \tau_{i_{p}} \hat{D}_{p}\right) \phi_{0}\left(x_{0}, \hat{x}, x\right)=0 \quad(1 \leqq i \leqq g) .
$$

From $\phi_{0}\left(x_{0}, \hat{x}, x\right)=\rho\left(x_{0}, 0,0\right) \phi_{0}(0, \hat{x}, x)=\exp \left(-2 \pi n \sqrt{-1} x_{0}\right) \phi_{0}(0, \hat{x}, x)$ we see that $\phi_{0}\left(x_{0}, \hat{x}, x\right)$ satisfies the conditions in Lemma 2.1, and thus

$$
\phi_{0}\left(x_{0}, \hat{x}, x\right)=\exp \left(\pi n \sqrt{-1}\left(x \tau^{t} x+2 \hat{x}^{t} x-2 x_{0}\right)\right) \sum_{b \in \mathbf{Z}^{8} / n Z^{8}} \alpha_{b} \vartheta(n)\left[\begin{array}{c}
b / n \\
0
\end{array}\right](\tau \mid \hat{x}+x \tau)
$$

with constants $\alpha_{b}$. Hence

$$
\phi_{0}\left(x_{0}, \hat{x}, x\right) \in \underset{b \in \mathbb{Z}^{8 / n Z^{g}}}{\bigoplus} H^{(n)}\left[\begin{array}{c}
b / n \\
0
\end{array}\right]=H^{(n)} .
$$

On the other hand $W$ is spanned by $\hat{D}^{j} \phi_{0}\left(j \in Z_{\geqq 0}^{g}\right)$, and thu;

$$
W \subset \underset{b \in Z^{8 / n Z^{8}}}{\oplus} \boldsymbol{H}^{(n)}\left[\begin{array}{c}
b / n \\
0
\end{array}\right] \text {. }
$$

From the relation

$$
\begin{gathered}
\phi_{j}^{(n)}\left[\begin{array}{c}
a / n \\
0
\end{array}\right]\left(\tau \mid x_{0}, \hat{x}+\frac{\hat{a}}{n}, x\right) \\
=\exp \left(2 \pi \sqrt{-1}\left(\hat{a}^{t} x+\frac{1}{n} \hat{a}^{t} a\right) \phi_{j}^{(n)}\left[\begin{array}{c}
a / n \\
0
\end{array}\right]\left(\tau \mid x_{0}, \hat{x}, x\right)\right. \\
\left(\hat{a} \in \boldsymbol{Z}^{g} / n \boldsymbol{Z}^{g}, j \in \boldsymbol{Z}_{\geqq 00}^{g}\right)
\end{gathered}
$$

we observe that an element $\phi$ in

$$
\underset{b \in \mathbf{Z}^{8} / n \mathbf{Z}^{g}}{\oplus} \boldsymbol{H}^{(n)}\left[\begin{array}{c}
b / n \\
0
\end{array}\right] \text { belongs to } \boldsymbol{H}^{(n)}\left[\begin{array}{c}
a / n \\
0
\end{array}\right]
$$

if and only if 


$$
\phi\left(x_{0}, \hat{x}+\frac{\hat{a}}{n}, x\right)=\exp \left(2 \pi \sqrt{-1}\left(\hat{a}^{t} x+\frac{1}{n} \hat{a}^{t} a\right)\right) \phi\left(x_{0}, \hat{x}, x\right)
$$

$\left(\hat{a} \in Z^{g} / n Z^{g}\right)$.

On the other hand $\oplus_{b \in \boldsymbol{Z}^{g} / n \boldsymbol{Z}^{g}} \boldsymbol{H}^{(n)}\left[\begin{array}{c}b / n \\ 0\end{array}\right]$ are independent on the choice of $\tau$, hence each $\boldsymbol{H}^{(n)}\left[\begin{array}{c}a / n \\ 0\end{array}\right]$ is independent on the choice of $\tau$. From (1.18) we have

$$
\boldsymbol{H}^{(n)}\left[\begin{array}{c}
a / n \\
0
\end{array}\right]=\exp \left(2 \pi \sqrt{-1} a^{t} \hat{x}\right) \boldsymbol{H}^{(n)}\left[\begin{array}{l}
0 \\
0
\end{array}\right]
$$

Corollary 2.2.1. For a non-zero integer $n, \rho_{n}$ means the irreducible unitary representation such that

$$
\rho_{n}\left(y_{0}, 0,0\right) \phi=\exp \left(-2 \pi n \sqrt{-1} y_{0}\right) \phi \quad\left(y_{0} \in \boldsymbol{R}\right),
$$

then the multiplicity $m_{\rho: \rho_{n}}$ of $\rho_{n}$ in $\rho$ is given by

$$
m_{\rho: \rho_{n}}=|n|^{g} \text {. }
$$

Proof. The space of theta functions of level $n$ is a vector space of dimension $n^{g}$, hence by virtue of Theorem 2.1 we have (2.3).

2.2. Using Hermitian polynomials we shall first construct an orthogonal basis of $L^{2}\left(H_{2 g+1}(Z) \backslash H_{2 g+1}(R)\right)$ associating with $\tau$, and define a natural unitary representation of $S p_{2 g}(R)$ on $L^{2}\left(H_{2 g+1}(Z) \backslash H_{2 g+1}(R)\right)$.

Hermitian polynomials $H_{n}(v)$ in one variable $v$ are defined by the generating function

$$
\exp \left(-\left(s^{2}-2 s v\right)\right)=\sum_{n=0}^{\infty} \frac{s^{n}}{n !} H_{n}(v)
$$

which satisfy the orthogonal relation:

$$
\int_{-\infty}^{\infty} H_{n}(v) H_{m}(v) e^{-v^{2}} d v=\left\{\begin{array}{cc}
2^{n} n ! \sqrt{\pi} & (n=m) \\
0 & (n \neq m) .
\end{array}\right.
$$

For $j=\left(j_{1}, \cdots, j_{g}\right) \in Z_{\geqq 0}^{g}$ and $x=\left(x_{1}, \cdots, x_{g}\right)$ we denote

$$
H_{j}(x)=H_{j_{1}}\left(x_{1}\right) \cdots H_{j_{g}}\left(x_{g}\right)
$$

then Hermitian polynomials in many variables satisfy the orthogonal relation 


$$
\int_{\boldsymbol{R}^{g}} H_{j}(x) H_{k}(x) \exp \left(-x^{t} x\right) d x=\left\{\begin{array}{cc}
2^{|j|} j ! \pi^{g / 2} & (j=k) \\
0 & (j \neq k) .
\end{array}\right.
$$

Since $\tau^{\prime \prime}=(1 / 2 \sqrt{-1})(\tau-\bar{\tau})$ is positive definite, we can define the unique square root $\sqrt{\tau^{\prime \prime}}$. The composite functions

$$
H_{j}\left(x \sqrt{2 \pi \tau^{\prime \prime}}\right) \quad\left(j \in Z_{\geqq 0}^{g}\right)
$$

satisfy the orthogonal relation:

$$
\begin{gathered}
\int_{R^{g}} H_{j}\left(x \sqrt{2 \pi \tau^{\prime \prime}}\right) H_{k}\left(x \sqrt{2 \pi \tau^{\prime \prime}}\right) \exp \left(-2 \pi n x \tau^{\prime \prime} x\right) d x \\
=\left\{\begin{array}{cl}
\frac{2^{|j|} j !}{2^{g / 2} \sqrt{\operatorname{det} \tau^{\prime \prime}}} & (j=k) \\
0 & (j \neq k) .
\end{array}\right.
\end{gathered}
$$

Theorem 2.2. Putting

$$
\begin{gathered}
H_{j}^{(n)}\left[\begin{array}{c}
a / n \\
0
\end{array}\right]\left(\tau \mid x_{0}, \hat{x}, x\right) \\
=\frac{2^{g / 4}\left(\operatorname{det} \tau^{\prime \prime}\right)^{1 / 4}}{2^{|j| / 2} \sqrt{j !}} \exp \left(-2 \pi n \sqrt{-1} x_{0}\right) \sum_{\ell \in Z^{g}} H_{j}\left(\left(x+\ell+\frac{a}{n}\right) \sqrt{2 \pi \tau^{\prime \prime}}\right) \\
\cdot \exp \left(\pi n \sqrt{-1}\left(\left(x+\ell+\frac{a}{n}\right) \tau^{t}\left(x+\ell+\frac{a}{n}\right)+2 \hat{x}^{t}\left(x+\ell+\frac{a}{n}\right)\right)\right. \\
\quad\left(a \in Z^{g} / n Z^{g}, j \in Z_{\geqq}^{g}, n \geqq 1\right),
\end{gathered}
$$

we obtain an orthonormal basis

$$
\left\{H_{j}^{(n)}\left[\begin{array}{c}
a / n \\
0
\end{array}\right]\left(\tau \mid x_{0}, \hat{x}, x\right) \mid j \in Z_{\geqq 0}^{g}\right\} \quad \text { of } \quad H^{(n)}\left[\begin{array}{c}
a / n \\
0
\end{array}\right] .
$$

Proof. From the orthogonal relation for $H_{j}\left(x \sqrt{2 \pi \tau^{\prime \prime}}\right)$ and Lemma 1.2 it follows the orthogonal relation

$$
\begin{aligned}
& \int_{H_{2 g+1}(\boldsymbol{Z}) \backslash H_{2}{ }_{+1}(\boldsymbol{R})} H_{j}^{(n)}\left[\begin{array}{c}
a / n \\
0
\end{array}\right] \overline{\left(\tau \mid x_{0}, \hat{x}, x\right)} H_{k}^{n}\left[\begin{array}{c}
a / n \\
0
\end{array}\right]\left(\tau \mid x_{0}, \hat{x}, x\right) d x_{0} d \hat{x} d x \\
& = \begin{cases}1 & (j=k) \\
0 & (j \neq k) .\end{cases}
\end{aligned}
$$

Corollary 2.2.1. $\quad L^{2}\left(H_{2 g+1}(Z) \backslash H_{2 g+1}(R)\right)$ has an orthonormal basis

$$
\begin{aligned}
& \left\{H_{j}^{(n)}\left[\begin{array}{c}
a / n \\
0
\end{array}\right]\left(\tau \mid x_{0}, \hat{x}, x\right), \overline{H_{j}^{(n)}\left[\begin{array}{c}
a / n \\
0
\end{array}\right]\left(\tau \mid x_{0}, \hat{x}, x\right)}\right. \\
& \exp \left(2 \pi \sqrt{-1}\left(\hat{k}^{t} \hat{x}+k^{t} x\right)\right) \mid a \in Z^{g} / n Z^{g}, \\
& \left.\left.j \in Z_{\geqq 0}^{g}, n \geqq 1,(\hat{k}, k) \in Z^{g} \times Z^{g}\right)\right\},
\end{aligned}
$$


such that

$$
\left.H_{\jmath}^{(n)}\left[\begin{array}{c}
a / n \\
0
\end{array}\right]\left(\tau \mid x_{0}, \hat{x}, x\right)=\exp (2 \pi \sqrt{-1} \hat{a}) H_{j}^{(n)}\left[\begin{array}{l}
0 \\
0
\end{array}\right]\left(\tau \mid x_{0}, \hat{x}, x\right) \circ\left(0,0, \frac{a}{n}\right)\right) .
$$

\section{REFERENCES}

[1] V. Bargmann, On a Hilbert space of analytic functions and an associated integral transform I, Comm. Pure Appl. Math., 14 (1961), 187-214.

[2] J. Igusa, Theta functions, Springer, 1972.

[ 3 ] H. Morikawa, A decomposition theorem on differential polynomials of theta functions, Nagoya Math. J., 96 (1984), 113-126.

[4] I. Satake, Fock representations and theta functions, Ann. Math. Studies, 66, Princeton Univ. Press (1971), 393-405.

Department of Mathematics

Faculty of Science

Nagoya University

Chikusa-ku, Nagoya 464

Japan 